\title{
Sensitization of primary cultures from rat dorsal root ganglia with lipopolysaccharide (LPS) requires a robust inflammatory response
}

\author{
Franz Nürnberger ${ }^{1}$. Stephan Leisengang ${ }^{1}$. Daniela Ott ${ }^{1}$. Jolanta Murgott ${ }^{1} \cdot$ Rüdiger Gerstberger $^{1}$. \\ Christoph Rummel ${ }^{1}$ Joachim Roth ${ }^{1}$ (i)
}

Received: 15 July 2021 / Revised: 8 December 2021 / Accepted: 10 December 2021 / Published online: 23 December 2021

(c) The Author(s) 2021

\begin{abstract}
Objective We investigated whether it is possible to induce a state of "LPS-sensitization" in neurons of primary cultures from rat dorsal root ganglia by pre-treatment with ultra-low doses of LPS.

Methods DRG primary cultures were pre-treated with low to ultra-low doses of LPS $(0.001-0.1 \mu \mathrm{g} / \mathrm{ml})$ for $18 \mathrm{~h}$, followed by a short-term stimulation with a higher LPS-dose $(10 \mu \mathrm{g} / \mathrm{ml}$ for $2 \mathrm{~h})$. TNF- $\alpha$ in the supernatants was measured as a sensitive read out. Using the fura-2 $340 / 380 \mathrm{~nm}$ ratio imaging technique, we further investigated the capsaicin-evoked $\mathrm{Ca}^{2+}$-signals in neurons from DRG, which were pre-treated with a wide range of LPS-doses.

Results Release of TNF- $\alpha$ evoked by stimulation with $10 \mu \mathrm{g} / \mathrm{ml}$ LPS into the supernatant was not significantly modified by pre-exposure to low to ultra-low LPS-doses. Capsaicin-evoked $\mathrm{Ca}^{2+}$-signals were significantly enhanced by pre-treatment with LPS doses being above a certain threshold.

Conclusion Ultra-low doses of LPS, which per se do not evoke a detectable inflammatory response, are not sufficient to sensitize neurons $\left(\mathrm{Ca}^{2+}\right.$-responses) and glial elements (TNF- $\alpha$-responses) of the primary afferent somatosensory system.
\end{abstract}

Keywords LPS sensitization - Dorsal root ganglia $\cdot$ Mixed neuro-glial cultures $\cdot$ Inflammation $\cdot$ Cytokines $\cdot$ Capsaicin $\cdot$ $\mathrm{Ca}^{2+}$-imaging

\section{Introduction}

Pre-exposure of macrophages to LPS causes either tolerance, meaning that the responses to a second LPS-challenge are strongly attenuated, or priming, an elevated response to a second hit of LPS [1]. Cells or animals become LPStolerant when a first challenge with LPS causes a robust inflammatory response. Priming or sensitization is induced by ultra-low LPS-doses, that per se will not evoke substantial formation and release of pro-inflammatory cytokines. A state of LPS-tolerance can also be evoked in structures of the peripheral or central nervous system [2]. Whether or not

Responsible Editor: John Di Battista.

Joachim Roth

joachim.roth@vetmed.uni-giessen.de

Department of Veterinary-Physiology and -Biochemistry, Justus Liebig University Giessen, Frankfurter Strasse 100, 35392 Giessen, Germany
LPS-sensitization will occur in a given neuroglial structure, especially in dorsal root ganglia (DRG), has not yet been investigated. The central goals of this study can, therefore, be summarized as follows: we first tried to determine ultralow LPS-doses for a long-term stimulation of DRG cultures for $18 \mathrm{~h}$, which per se did not cause elevations of TNF- $\alpha$ in the supernatants but an enhanced production of this cytokine by a second hit with a high LPS-dose ("sensitization"). We further tested the effects of the presence of ultra-low, moderate and high LPS-doses on the capsaicin-evoked responses of neurons from rat DRG.

\section{Materials and methods}

See supplementary material. 


\section{Results}

Cultivation of DRG primary cultures in presence of various doses of LPS was accompanied by a dose dependent rise of TNF- $\alpha$ in the supernatants (Fig. 1A). The lowest LPS-doses used in this experiment $(0.01$ and $0.001 \mu \mathrm{g} /$ $\mathrm{ml}$ ) did not evoke a significant increase of TNF- $\alpha$ in the supernatants. To test whether primary DRG cultures were sensitized to a subsequent hit with a high LPS dose, cultures were stimulated with $10 \mu \mathrm{g} / \mathrm{ml}$ LPS for $2 \mathrm{~h}$ after pre-exposure with $0.1,0.01$ or $0.001 \mu \mathrm{g} / \mathrm{ml}$ LPS for $18 \mathrm{~h}$ (Fig. 1B). The slight LPS-induced $(10 \mu \mathrm{g} / \mathrm{ml})$ increase of TNF- $\alpha$ in supernatants of cells pre-treated with $0.001 \mu \mathrm{g} /$ ml LPS was not significant.

DRG primary cultures were incubated in presence of PBS or LPS at various doses $(0.001,0.01,0.1$ or $1 \mu \mathrm{g} /$ $\mathrm{ml}$ ) for $18 \mathrm{~h}$. Thereafter, the strength of stimulus-induced $\mathrm{Ca}^{2+}$-signals of DRG-neurons was evaluated [3]. Capsaicin,
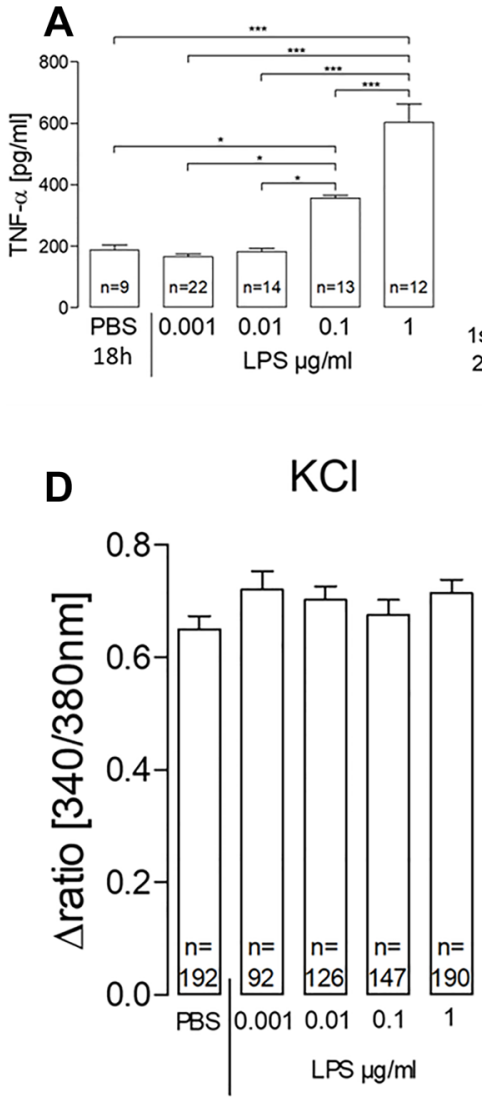
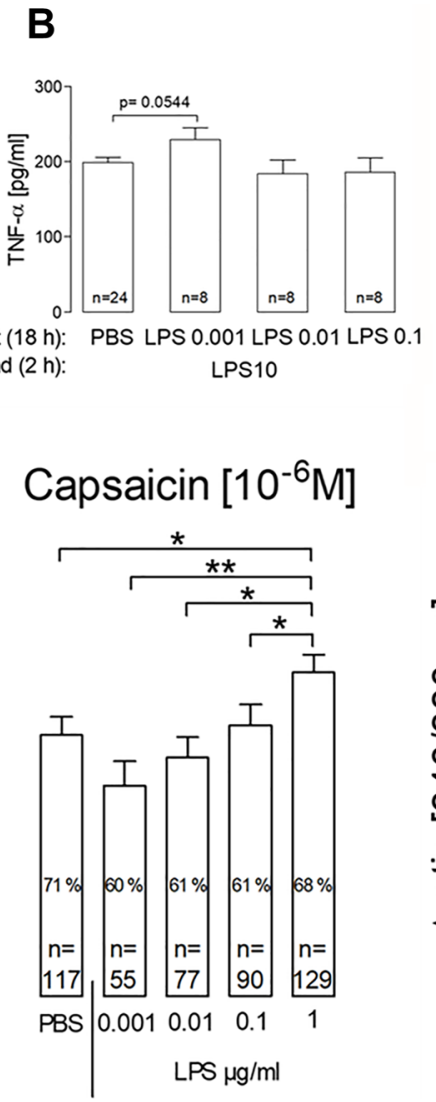

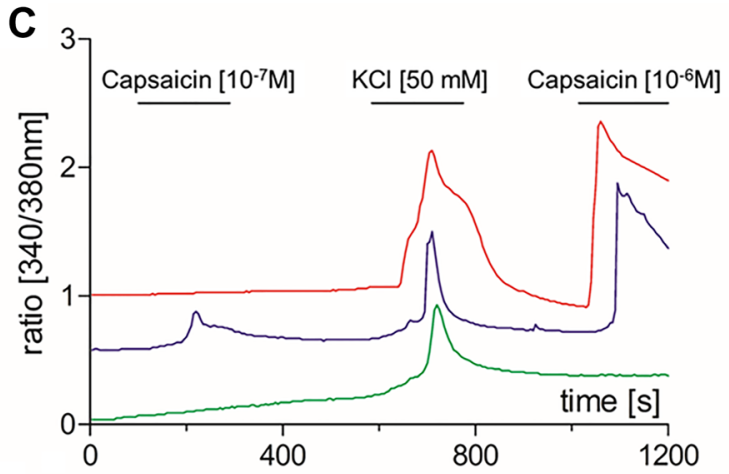

E

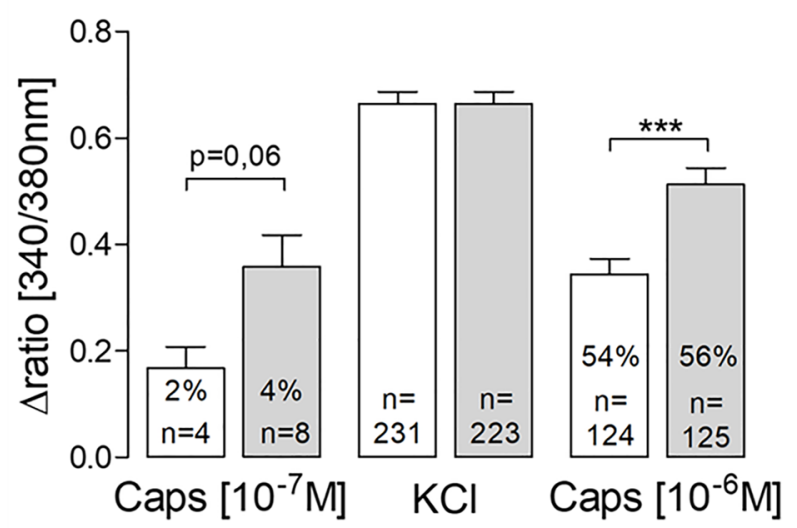

Fig. 1 A, B TNF- $\alpha$ release after incubation with different doses of LPS and PBS: DRG primary cultures were incubated with different doses of LPS $(0.001,0.01,0.1,1 \mu \mathrm{g} / \mathrm{ml})$ for $18 \mathrm{~h}$ or PBS as control (A) for subsequent measurement of TNF- $\alpha$ in the supernatants. In a second series of experiments (B), DRG primary cultures were pre incubated with different doses $(0.001,0.01,0.1 \mu \mathrm{g} / \mathrm{ml})$ for $18 \mathrm{~h}$ as well as PBS as control followed by a second stimulation with a high LPS dose $(10 \mu \mathrm{g} / \mathrm{ml})$ for $2 \mathrm{~h}$. Pre-incubation with $0.001 \mu \mathrm{g} / \mathrm{ml}$ followed by a stimulation with a high LPS dose showed a tendency of a higher TNF- $\alpha$ release into supernatants compared to control. Each column represents the mean \pm SEM of $n$ samples and four different experiments. One-way ANOVA followed by a Newman-Keuls multiple comparison test was performed for statistical analysis. The graphical depiction of the $p$ values were illustrated as follows: $* * * p<0.001 ; * * p<0.01 ; * p<0.05$; D DRG primary cells were stimulated with different doses $(0.001,0.01,0.1,1 \mu \mathrm{g} / \mathrm{ml})$ of LPS for $18 \mathrm{~h}$ and PBS as control. $\mathrm{KCl}$ served as vitality control for DRG neurons. $\Delta$ ratio $[340 / 380 \mathrm{~nm}]$ represents the mean \pm SEM increase of intracellular calcium $\left[\mathrm{Ca}^{2+}\right]_{\mathrm{i}}$ of $n$ cells of six different preparations.
Percentages represent the numbers of responsive cells to a distinct stimulus, e.g., capsaicin compared to all vital neurons $(\mathrm{KCl})$. Statistical analysis of $\mathrm{KCl}$ or capsaicin responses was performed using a one-way ANOVA followed by a Newman-Keuls multiple comparison test. $p$ values were represented as follows: $* * * p<0.001$; $* * p<0.01 ; * p<0.05 ; \mathbf{C}, \mathbf{E}$ Prior to the experiments DRG primary cultures were incubated with LPS $(1 \mu \mathrm{g} / \mathrm{ml}$ for $18 \mathrm{~h})$ or PBS $(18 \mathrm{~h})$. After incubation different stimuli (capsaicin $\left[10^{-7} \mathrm{M}\right], \mathrm{KCl}$ and capsaicin $\left.\left[10^{-6} \mathrm{M}\right]\right)$ were applied to investigate neuronal responses. $\mathbf{C}$ Examples of tracings recorded from three DRG neurons: one neuron responding to $\mathrm{KCl}[50 \mathrm{mM}]$ only (green), one responding to $\mathrm{KCl}$ and capsaicin $\left[10^{-6} \mathrm{M}\right]$ (red) and one responding to all three stimuli $\mathrm{KCl}$, capsaicin $\left[10^{-6} \mathrm{M}\right.$ and $\left.10^{-7} \mathrm{M}\right]$ (blue). E Depicts $\Delta$ ratio $[340 / 380 \mathrm{~nm}]$ fluorescence values as a measurement of $\left[\mathrm{Ca}^{2+}\right]$. Columns represent the mean \pm SEM increase of intracellular calcium $\left[\mathrm{Ca}^{2+}\right]_{i}$ of $n$ cells from 4 different preparations. Statistical analysis between the PBS and LPS group was performed using an unpaired $t$ test. $* * * p<0.001$ (color figure online) 
at a dose of $10^{-6} \mathrm{M}$, evoked pronounced $\mathrm{Ca}^{2+}$-responses in $60-70 \%$ of neurons from all groups (Fig. 1D).

The responses of neurons to the depolarizing $\mathrm{KCl}$-solution (vitality-test) was similar in all groups investigated. A significant enhancement of the strength of capsaicin-induced $\mathrm{Ca}^{2+}$-signals was exclusively determined in the group, which was pre-treated with the highest LPS-dose $(1 \mu \mathrm{g} / \mathrm{ml}$, Fig. 1D), the same dose, which evoked a profound increase of TNF- $\alpha$ in the supernatants (Fig. 1A). A sensitization of DRG neurons to a nociceptive stimulus (capsaicin) [4] thus was not achieved by pre-treatment with very low LPS-dose, which per se did not evoke an increase of TNF- $\alpha$ production.

We finally tested whether DRG neurons might also show enhanced responses to the threshold-dose of capsaicin. We determined that not a single DRG neuron showed $\mathrm{Ca}^{2+}$-responses to capsaicin at doses $10^{-9}$ and $10^{-8} \mathrm{M}$. Stimulation with $10^{-7} \mathrm{M}$ capsaicin evoked $\mathrm{Ca}^{2+}$-signals just in single neurons. This dose was, therefore, defined as the threshold dose (Fig. 1C).

Just 4 out of 231 DRG neurons (about 2\%) responded to $10^{-7} \mathrm{M}$ capsaicin. In cultures pre-treated with $1 \mu \mathrm{g} / \mathrm{ml}$ LPS 8 out of 223 neurons (about 4\%) were responsive to the capsaicin threshold dose (Fig. 1D). Vitality of neurons (responses to $\mathrm{KCl}$ ) was identical in both groups. Again, the responses of DRG neurons to the effective capsaicin-dose of $10^{-6} \mathrm{M}$ was significantly higher in neurons pre-exposed to $1 \mu \mathrm{g} / \mathrm{ml}$ LPS.

\section{Discussion}

Priming of macrophages with a sub-threshold dose of LPS resulted in enhanced release of TNF- $\alpha$ to a second stimulation with a higher LPS-dose [5]. This effect seems to be the basic mechanism for the phenomenon of LPS-sensitization. We aimed to define experimental conditions, which should mimic such an effect in mixed neuro-glial primary cultures from rat DRG. With regard to the formation and release of TNF- $\alpha$, the outcome was not as clear as we expected. When pre-treated with an ultra-low LPS-dose, an enhanced TNF- $\alpha$ response to a subsequent stimulation with a high LPS-dose was hardly detectable. Still, there is evidence for a sensitization of capsaicin-responsive (nociceptive) sensory neurons from thoracic DRG with LPS resulting in hypersecretion in the upper airways [6]. Therefore, we tested the effects of cultivation of DRG primary cultures in presence of various doses of LPS on the strength of capsaicin-evoked $\mathrm{Ca}^{2+}$-signals. To evoke enhanced capsaicin responses, the presence of an amount of LPS in the culture medium is required, which induces a robust release of TNF- $\alpha$ into the supernatant. Sub-threshold doses of LPS failed to induce such an effect. This means that a given inflammatory insult has to reach a certain threshold to cause a sensitization of peripheral nociceptors finally resulting in the manifestation of inflammatory pain. Our observation that ultra-low doses of LPS failed to evoke sensitization of DRG nociceptive neurons (capsaicin-responses) and the mixed neuroglial culture (formation of TNF- $\alpha$ ) can possibly be explained by the fact that just $2 \%$ of all cells of DRG cultures are macrophages [2]. Cells from the macrophage-monocyte-lineage seem to be critical for sensitization to LPS, also in structures of the nervous system [7, 8]. Future studies should, therefore, employ central nervous structures including a higher percentage of cells from the macrophage-monocyte-lineage to investigate the phenomenon of LPS-sensitization in mixed neuroglial tissue.

Supplementary Information The online version contains supplementary material available at https://doi.org/10.1007/s00011-021-01534-2.

Funding Open Access funding enabled and organized by Projekt DEAL. Funding was provided by Justus Liebig Universität Gießen.

Open Access This article is licensed under a Creative Commons Attribution 4.0 International License, which permits use, sharing, adaptation, distribution and reproduction in any medium or format, as long as you give appropriate credit to the original author(s) and the source, provide a link to the Creative Commons licence, and indicate if changes were made. The images or other third party material in this article are included in the article's Creative Commons licence, unless indicated otherwise in a credit line to the material. If material is not included in the article's Creative Commons licence and your intended use is not permitted by statutory regulation or exceeds the permitted use, you will need to obtain permission directly from the copyright holder. To view a copy of this licence, visit http://creativecommons.org/licenses/by/4.0/.

\section{References}

1. Morris MC, Gilliam EA, Li L. Innate immune programing by endotoxin and its pathological consequences. Front Immunol. 2014;5:680.

2. Nürnberger F, Leisengang S, Ott D, Murgott J, Gerstberger R, Rummel C, Roth J. Manifestation of lipopolysaccharide-induced tolerance in neuro-glial primary cultures of the rat afferent somatosensory system. Inflamm Res. 2021;70(4):429-44.

3. Leisengang S, Ott D, Murgott J, Gerstberger R, Rummel C, Roth J. Primary cultures from rat dorsal root ganglia: responses of neurons and glial cells to somatosensory or inflammatory stimulation. Neuroscience. 2018;394:1-13.

4. Garami A, Shimansky YP, Pakai E, Oliveira DL, Gawa NR, Romanovsky AA. Contributions of different modes of TRPV1 activation to TRPV1 antagonist-induced hyperthermia. J Neurosci. 2010;30:1435-40.

5. Zhang X, Morrison DC. Lipopolysaccharide-induced selective priming effects on tumor necrosis factor alpha and nitric oxide production in mouse peritoneal macrophages. J Exp Med. 1993; 177:511-6.

6. Nair M, Jagadeeshan S, Katselis G, Luan X, Momeni Z, HenaoRomero N, Chumala P, Tam JS, Yamamoto Y, Ianowski JP, Campanucci VA. Lipopolysaccharides induce a RAGE-mediated sensitization of sensory neurons and fluid hypersecretion in the upper airways. Sci Rep. 2012;11:8336. 
7. Lajqi T, Lang G-P, Haas F, Williams DL, Hudalla H, Bauer M, Groth M, Wetzker R, Bauer R. Memory-like inflammatory responses of microglia to rising doses of LPS: key role of PI3K $\gamma$. Front Immunol. 2019;10:2492.

8. Neher JJ, Cunningham C. Priming microglia for innate immune memory in the brain. Trends Immunol. 2019;40:358-74.
Publisher's Note Springer Nature remains neutral with regard to jurisdictional claims in published maps and institutional affiliations. 